MATHEMATICS OF COMPUTATION

Volume 77, Number 261, January 2008, Pages 379-395

S 0025-5718(07)02025-X

Article electronically published on June 20, 2007

\title{
A REGULARIZED PROJECTION METHOD FOR COMPLEMENTARITY PROBLEMS WITH NON-LIPSCHITZIAN FUNCTIONS
}

\author{
GOETZ ALEFELD AND XIAOJUN CHEN
}

\begin{abstract}
We consider complementarity problems involving functions which are not Lipschitz continuous at the origin. Such problems arise from the numerical solution for differential equations with non-Lipschitzian continuity, e.g. reaction and diffusion problems. We propose a regularized projection method to find an approximate solution with an estimation of the error for the nonLipschitzian complementarity problems. We prove that the projection method globally and linearly converges to a solution of a regularized problem with any regularization parameter. Moreover, we give error bounds for a computed solution of the non-Lipschitzian problem. Numerical examples are presented to demonstrate the efficiency of the method and error bounds.
\end{abstract}

\section{INTRODUCTION}

Let $F: R^{n} \rightarrow R^{n}$ be defined by

$$
F(x)=M x+\phi(x),
$$

where $M$ is an $n \times n$ matrix and $\phi: R^{n} \rightarrow R^{n}$ is a monotonically increasing and continuous diagonal function, but not Lipschitz continuous at the origin. A function $g: R^{n} \rightarrow R^{n}$ is called a monotonically increasing diagonal function if $g_{i}(x)=g_{i}\left(x_{i}\right)$, and

$$
\left(g_{i}\left(x_{i}\right)-g_{i}\left(y_{i}\right)\right)\left(x_{i}-y_{i}\right) \geq 0, \quad i=1,2, \ldots, n .
$$

A function $g: R^{n} \rightarrow R^{n}$ is said to be Lipschitz continuous at $x$ if there exist an open set $\mathcal{D} \subset R^{n}, x \in \mathcal{D}$, and a constant $\kappa$ such that for all $y \in \mathcal{D}$

$$
\|g(x)-g(y)\| \leq \kappa\|x-y\| .
$$

See [5]. In this paper, we consider the nonlinear complementarity problem

$$
x \geq 0, \quad F(x) \geq 0, \quad x^{T} F(x)=0,
$$

and denote it by $\operatorname{NCP}(F)$. Such a non-Lipschitzian NCP arises from various applications. For instance, reaction and diffusion problems which can be modelled as free boundary problems.

Received by the editor June 8, 2006.

2000 Mathematics Subject Classification. Primary 90C33, 65G20.

Key words and phrases. Complementarity problems, non-Lipschitzian continuity, regularization, projection, error bounds.

(C)2007 American Mathematical Society 
Example 1.1 (2]). Let $\Omega$ be a bounded open set in $R^{2}$ with Lipschitz boundary $\partial \Omega$. Given two positive numbers $\lambda$ and $p \in(0,1)$, consider the free boundary problem

$$
\begin{array}{cl}
-\triangle u+\lambda u^{p}=0 & \text { in } \Omega_{+}, \\
u=0 & \text { in } \Omega_{0}, \\
u=|\nabla u|=0 & \text { on } \Gamma, \\
u=1 & \text { on } \partial \Omega
\end{array}
$$

where $\Omega_{+}=\{z \in \Omega \mid u(z)>0\}, \Omega_{0}=\{z \in \Omega \mid u(z)=0\}$, and $\Gamma=\partial \Omega_{0}=$ $\partial \Omega_{+} \cap \Omega$ are unknown. Using finite element approximation or finite difference approximation, we obtain a nonlinear complementarity problem with $F(x)=M x+$ $\phi(x)$, where

$$
\phi(x)=E \max \left(0, x^{p}\right)+q .
$$

Here $E$ is an $n \times n$ diagonal matrix with positive diagonals and $q$ is a vector in $R^{n}$. The function $\phi$ is a monotonically increasing diagonal function. However, since $p \in(0,1), \phi$ is not differentiable at the origin, and $\phi_{i}^{\prime}\left(x_{i}\right) \rightarrow \infty$ as $x_{i} \downarrow 0$.

The $\operatorname{NCP}(F)$ can be reformulated as a system of nonsmooth equations

$$
H(x):=\min (x, F(x))=0 .
$$

A number of algorithms for solving NCP have been developed based on the reformulation (1.2). See [6]. However, most algorithms require the involved function $F$ to be Lipschitz continuous. For instance, smoothing Newton-methods, semismooth Newton-methods and generalized Jacobian methods assume that $F$ is continuously differentiable in order to use the generalized Jacobian of $H$. The Rademacher theorem states that a locally Lipschitzian function in $R^{n}$ is almost everywhere differentiable. If $F$ is continuously differentiable in $R^{n}$, then $H$ is locally Lipschitz continuous in $R^{n}$. By the Rademacher theorem, the Clarke generalized Jacobian of $H$ can be defined by

$$
\partial H(x)=\operatorname{co}\left\{\lim _{\substack{x^{k} \rightarrow x \\ x^{k} \in D_{H}}} H\left(x^{k}\right)\right\}
$$

where $D_{H}$ denotes the set of points at which $H$ is differentiable and co denotes the convex hull. On the other hand, some numerical methods for nonlinear complementarity problems have nice global convergence properties for $F$ being a monotone function [6, 11], that is, $(F(x)-F(y))^{T}(x-y) \geq 0$, for $x, y \in R^{n}$.

Without Lipschitzian continuity and monotonicity, it seems hard to find an existing efficient method for solving the $\operatorname{NCP}(F)$. In this paper, we propose a regularized splitting method for solving the $\operatorname{NCP}(F)$ without assuming Lipschitzian continuity and monotonicity. Moreover, we give error bounds to verify accuracy of a computed solution of the $\operatorname{NCP}(F)$. In Section 2, for a given $\epsilon>0$, we define a regularization NCP which has a unique solution whose every element is not less than $\epsilon$. We prove that the sequence of the solutions of regularization problems with $\epsilon_{k}$ converges to the solution of the $\operatorname{NCP}(F)$ as $\epsilon_{k} \rightarrow 0$. In Section 3, we give error bounds for the non-Lipschtzian $\mathrm{NCP}(F)$ and its regularization problems with $M$ being a P-matrix. In Section 4, based on the error bounds, we present a projection method for solving the regularization problem which includes the Jacobi-type method, Gauss-Seidel-type method and SOR-type method as special cases. We 
prove that the projection method is globally and linearly convergent if $M$ is an H-matrix with positive diagonals.

We list some definitions and notation used in this paper.

An $n \times n$ matrix $A=\left(a_{i j}\right)$ is called a $\mathrm{P}$-matrix $\left(\mathrm{P}_{0}\right.$-matrix $)$ if all principal minors of $A$ are positive (nonnegative).

$A$ is called an $\mathrm{R}_{0}$-matrix, if the linear complementarity problem $x \geq 0, A x \geq$ $0, x^{T}(A x)=0$ has the zero vector as its unique solution.

$A$ is called an M-matrix, if $A^{-1} \geq 0$ and $a_{i j} \leq 0(i \neq j)$ for $i, j=1,2, \ldots, n$.

$A$ is called an H-matrix, if its comparison matrix $\tilde{A}=\left(\tilde{a}_{i j}\right)$ is an M-matrix, where

$$
\tilde{a}_{i j}=\left\{\begin{array}{rr}
\left|a_{i j}\right|, \quad i=j, \\
-\left|a_{i j}\right|, \quad i \neq j,
\end{array} \quad i, j=1,2, \ldots, n .\right.
$$

A function $F: R^{n} \rightarrow R^{n}$ is called a uniformly P-function, if there is a constant $\gamma>0$ such that

$$
\max _{1 \leq i \leq n}\left(x_{i}-y_{i}\right)\left(F_{i}(x)-F_{i}(y)\right) \geq \gamma\|x-y\|_{2}^{2} .
$$

A diagonal matrix $W$ whose diagonal elements are defined by a vector $w=$ $\left(w_{1}, \ldots, w_{n}\right)^{T}$ is written as $W=\operatorname{diag}\left(w_{i}\right)$. Let $[a]$ denote an interval vector (matrix), and $\underline{a}$ and $\bar{a}$ denote the lower bound and upper bound of $[a]$, respectively, that is, $[a]=\{x \mid \underline{a} \leq x \leq \bar{a}\}$. For given two vectors $u, v(u \leq v)$ the mid function is defined by

$$
\operatorname{mid}(u, v, y)_{i}=\left\{\begin{array}{cc}
v_{i}, & v_{i}<y_{i}, \\
y_{i}, & u_{i} \leq y_{i} \leq v_{i}, \\
u_{i} . & u_{i}>y_{i} .
\end{array}\right.
$$

For a given interval vector $[a], \Pi_{[a]}(\cdot):=\operatorname{mid}(\underline{a}, \bar{a}, \cdot)$ is the Euclidean projector $\Pi$ onto the interval $[a]$. The nonnegative orthant is denoted by $R_{+}^{n}$. The max function $\max (0, \cdot)$ is the Euclidean projector $\Pi_{R_{+}^{n}}(\cdot)$ onto $R_{+}^{n}$.

\section{Regularization MEthod}

We consider a system of regularization equations of (1.2),

$$
H_{\epsilon}(x)=\min (x, M x+\phi(x))-\epsilon e=0,
$$

where $\epsilon$ is a positive number and $e$ is the $n$-dimensional vector whose elements are all 1 .

Lemma 2.1. Assume that $M$ is a P-matrix and $\phi$ is a monotonically increasing diagonal function. Then for any $\epsilon \geq 0$, the system of regularization equations (2.1) has a unique solution.

Proof. It is easy to see that (2.1) can be rewritten as

$$
H_{\epsilon}(x)=\min (x-\epsilon e, M x+\phi(x)-\epsilon e)=0 .
$$

Let $y=x-\epsilon e$. Then we can write (2.1) as an NCP in the following form:

$$
\min (y, M y+\epsilon(M-I) e+\phi(y+\epsilon e))=0 .
$$

Let us define

$$
F_{\epsilon}(y)=M y+\epsilon(M-I) e+\phi(y+\epsilon e) .
$$

Then for any $u, v \in R^{n}$, we have

$$
F_{\epsilon}(u)-F_{\epsilon}(v)=M(u-v)+\phi(u+\epsilon e)-\phi(v+\epsilon e) .
$$


Since $M$ is a $\mathrm{P}$-matrix, we have

$$
c(M):=\min _{\|x\|_{\infty}=1}\left\{\max _{1 \leq i \leq n} x_{i}(M x)_{i}\right\}>0 .
$$

See [4. Therefore, from $\phi$ being monotonically increasing, we obtain, for any $u, v \in R^{n}$,

$$
\begin{aligned}
& \max _{1 \leq i \leq n}\left(u_{i}-v_{i}\right)\left(F_{\epsilon}(u)-F_{\epsilon}(v)\right)_{i} \\
& =\max _{1 \leq i \leq n}\left\{\left(u_{i}-v_{i}\right)(M(u-v))_{i}+\left(u_{i}-v_{i}\right)(\phi(u+\epsilon e)-\phi(v+\epsilon e))_{i}\right\} \\
& \geq \max _{1 \leq i \leq n}\left(u_{i}-v_{i}\right)(M(u-v))_{i} \\
& \geq c(M)\|u-v\|_{\infty}^{2} \\
& \geq \frac{c(M)}{n}\|u-v\|_{2}^{2} .
\end{aligned}
$$

This implies that $F_{\epsilon}$ is a uniformly P-function. By Proposition 3.5.10 in [6], the $\operatorname{NCF}\left(F_{\epsilon}\right)$ has a unique solution, and hence the system of regularization equations (2.1) has a unique solution.

Let $\left\{\epsilon_{k}\right\}$ be a sequence of positive numbers, which satisfies

$$
\epsilon_{k} \geq \epsilon_{k+1}, k=0,1, \ldots, \quad \text { and } \quad \lim _{k \rightarrow \infty} \epsilon_{k}=0 .
$$

Let $x^{k}$ be the solution of $H_{\epsilon_{k}}(x)=0$. In the rest of this section, we study the convergence of the solution sequence $\left\{x^{k}\right\}$.

Let us denote the level set of the function $H(x)$ by

$$
\mathcal{S}(\mu)=\left\{x \in R^{n} \mid\|H(x)\| \leq \mu\right\}
$$

where $\mu \geq 0$.

Theorem 2.1. Assume that $M$ is a P-matrix and $\phi$ is a monotonically increasing and continuous diagonal function. Then the sequence $\left\{x^{k}\right\}$ converges to the unique solution of $H(x)=0$.

Proof. Following the proof of Lemma 2.1, we can show that $M x+\phi(x)$ is a uniformly P-function. Therefore, by Proposition 9.1.27 in [6], the level set $\mathcal{S}(\mu)$ is bounded for every $\mu \geq 0$.

Since $H_{\epsilon_{k}}\left(x^{k}\right)=0$ implies that

$$
\left\|H\left(x^{k}\right)\right\|=\epsilon_{k}\|e\| \leq \epsilon_{0}\|e\|,
$$

we deduce $\left\{x^{k}\right\} \subset \mathcal{S}\left(\epsilon_{0}\|e\|\right)$, and thus $\left\{x^{k}\right\}$ is bounded.

Let $\bar{x}$ be an accumulation point of $\left\{x^{k}\right\}$. From the equality

$$
H\left(x^{k}\right)=H\left(x^{k}\right)-H_{\epsilon_{k}}\left(x^{k}\right)=\epsilon_{k} e,
$$

and by the continuity of $H$, we find that for a subsequence, which we denote again by $\left\{x^{k}\right\}$,

$$
\|H(\bar{x})\|=\lim _{k \rightarrow \infty}\left\|H\left(x^{k}\right)\right\|=\lim _{k \rightarrow \infty} \epsilon_{k}\|e\|=0 .
$$

Hence $\bar{x}$ is a solution $x^{*}$ of $H(x)=0$. Since $x^{*}$ is the unique solution of $H(x)=0$, we deduce that the sequence $\left\{x^{k}\right\}$ converges to $x^{*}$. 
Theorem 2.2. Assume that $M$ is a $P_{0}$-matrix and $\phi$ is a monotonically increasing and continuous diagonal function. Assume there are positive constants $\Gamma$ and $\gamma$ such that for $x_{i} \geq \Gamma$,

$$
\frac{\phi_{i}\left(x_{i}\right)-\phi_{i}(0)}{x_{i}} \geq \gamma, \quad i=1,2, \ldots, n .
$$

If (2.1) with small $\epsilon_{k}>0$ has a solution $x^{k}$, then the sequence $\left\{x^{k}\right\}$ is bounded, and any accumulation point of $\left\{x^{k}\right\}$ is a solution of $H(x)=0$.

Proof. First we show that the sequence $\left\{x^{k}\right\}$ is bounded. Note that $H_{\epsilon_{k}}\left(x^{k}\right)=0$ implies that

$$
x^{k} \geq \epsilon_{k} e \quad \text { and } \quad M x^{k}+\phi\left(x^{k}\right) \geq \epsilon_{k} e .
$$

The sequences $\left\{x^{k}\right\}$ and $\left\{M x^{k}+\phi\left(x^{k}\right)\right\}$ are bounded below.

Assume that there is an $i$ such that $x_{i}^{k} \rightarrow \infty$. Let

$$
\mathcal{J}=\left\{i \mid x_{i}^{k} \rightarrow \infty\right\}
$$

and

$$
v^{k}=\frac{x^{k}}{\left\|x^{k}\right\|}
$$

Since $\left\{v^{k}\right\}$ is bounded, there is a convergent subsequence of $\left\{v^{k}\right\}$. By working with an appropriate subsequence of $\left\{v^{k}\right\}$ if necessary, we may assume without loss of generality that

$$
\lim _{k \rightarrow \infty} v^{k}=\bar{v} \geq 0 \quad \text { and } \quad\|\bar{v}\|=1 .
$$

Now we show that there is a matrix $N \in R^{n \times n}$ such that

$$
\lim _{k \rightarrow \infty} \min \left(\frac{x^{k}}{\left\|x^{k}\right\|}, M \frac{x^{k}}{\left\|x^{k}\right\|}+\frac{\phi\left(x^{k}\right)}{\left\|x^{k}\right\|}\right)=\min (\bar{v}, M \bar{v}+N \bar{v})=0 .
$$

Obviously, we have

$$
\lim _{k \rightarrow \infty} M \frac{x^{k}}{\left\|x^{k}\right\|}=M \bar{v}
$$

To prove (2.3), we only need to consider the limit of $\frac{\phi\left(x^{k}\right)}{\left\|x^{k}\right\|}$. First we observe that the limit of $\frac{\phi\left(x^{k}\right)}{\left\|x^{k}\right\|}$ exists, since for all $i \in \mathcal{J}$

$$
\left(M x^{k}+\phi\left(x^{k}\right)\right)_{i}=\epsilon_{k}, \quad \text { for large } k,
$$

which implies

$$
\lim _{k \rightarrow \infty} \frac{\phi_{i}\left(x_{i}^{k}\right)}{\left\|x^{k}\right\|}=\lim _{k \rightarrow \infty}\left(\frac{\epsilon_{k}}{\left\|x^{k}\right\|}-\left(M \frac{x^{k}}{\left\|x^{k}\right\|}\right)_{i}\right)=-(M \bar{v})_{i} .
$$

By the assumption (2.2), for large $k$, we have

$$
\frac{\phi_{i}\left(x_{i}^{k}\right)-\phi_{i}(0)}{x_{i}^{k}} \geq \gamma, \quad \text { for } i \in \mathcal{J} .
$$

Let

$$
\mathcal{J}_{1}=\left\{i \mid \bar{v}_{i}>0\right\} .
$$


It is easy to see that $\mathcal{J}_{1}$ is a nonempty subset of $\mathcal{J}$, since $\|\bar{v}\|=1$. Moreover, for $i \in \mathcal{J}_{1}$, by (2.4) and

$$
\begin{aligned}
\lim _{k \rightarrow \infty} \frac{\phi_{i}\left(x_{i}^{k}\right)}{\left\|x^{k}\right\|} & =\lim _{k \rightarrow \infty}\left(\frac{\phi_{i}\left(x_{i}^{k}\right)-\phi_{i}(0)}{x_{i}^{k}} \cdot \frac{x_{i}^{k}}{\left\|x^{k}\right\|}+\frac{\phi_{i}(0)}{x_{i}^{k}} \cdot \frac{x_{i}^{k}}{\left\|x^{k}\right\|}\right) \\
& =\lim _{k \rightarrow \infty} \frac{\phi_{i}\left(x_{i}^{k}\right)-\phi_{i}(0)}{x_{i}^{k}} \cdot \frac{x_{i}^{k}}{\left\|x^{k}\right\|}
\end{aligned}
$$

we find

$$
\lim _{k \rightarrow \infty} \frac{\phi_{i}\left(x_{i}^{k}\right)-\phi_{i}(0)}{x_{i}^{k}}=-\frac{(M \bar{v})_{i}}{\bar{v}_{i}}=: \alpha_{i} \geq \gamma
$$

Therefore, for $i \in \mathcal{J}_{1}$, we have

$$
\lim _{k \rightarrow \infty} \frac{\phi_{i}\left(x_{i}^{k}\right)}{\left\|x^{k}\right\|}=\alpha_{i} \bar{v}_{i}
$$

For $i \notin \mathcal{J},\left\{x_{i}^{k}\right\}$ is bounded. Since $\phi_{i}\left(x_{i}\right)$ is monotonically increasing and continuous, $\left\{\phi_{i}\left(x_{i}^{k}\right)\right\}$ is also bounded, and hence

$$
\lim _{k \rightarrow \infty} \frac{\phi_{i}\left(x_{i}^{k}\right)}{\left\|x^{k}\right\|}=0 .
$$

Therefore, we can define the $i$-th row of the matrix $N$ as follows:

$$
n_{i}^{T}=\left\{\begin{array}{ll}
\alpha_{i} e_{i}^{T}, & i \in \mathcal{J}_{1}, \\
-m_{i}^{T}, & i \in \mathcal{J} / \mathcal{J}_{1}, \\
0 e_{i}^{T}, & \text { otherwise },
\end{array} \quad i=1,2, \ldots, n,\right.
$$

where $e_{i}^{T}$ is the $i$-th row of the identity matrix and $m_{i}^{T}$ is the $i$-th row of $M$. Note that (2.3) implies that $\bar{v}$ is a solution of the linear complementarity problem

$$
x \geq 0, \quad(M+N) x \geq 0, \quad x^{T}(M+N) x=0 .
$$

Let $(M+N)_{J_{1}, J_{1}}$ and $N_{J_{1}, J_{1}}$ be the submatrices of $M+N$ and $N$ with rows and columns indexed by $\mathcal{J}_{1}$, respectively. Let $\bar{v}_{J_{1}}$ be the subvector of $\bar{v}$ with components indexed by $\mathcal{J}_{1}$. Note that for $i \notin \mathcal{J}_{1}, \bar{v}_{i}=0$. Hence (2.5) implies that $\bar{v}_{J_{1}}$ is a solution of

$$
x_{J_{1}} \geq 0, \quad(M+N)_{J_{1}, J_{1}} x_{J_{1}} \geq 0, \quad x_{J_{1}}^{T}(M+N)_{J_{1}, J_{1}} x_{J_{1}}=0 .
$$

Since $M$ is a $\mathrm{P}_{0}$-matrix and $N_{J_{1}, J_{1}}$ is a positive diagonal matrix, $(M+N)_{J_{1}, J_{1}}$ is a P-matrix, and thus (2.6) has the zero vector as its unique solution. This contradicts to $\left\|\bar{v}_{J_{1}}\right\|=1$. Therefore the sequence $\left\{x^{k}\right\}$ is bounded.

Let $\bar{x}$ be an accumulation point of $\left\{x^{k}\right\}$. From

$$
H_{\epsilon_{k}}\left(x^{k}\right)-H\left(x^{k}\right)=-\epsilon_{k} e=-H\left(x^{k}\right)
$$

and by the continuity of $H$, we have for a subsequence, which we denote again by $\left\{x^{k}\right\}$,

$$
\|H(\bar{x})\|=\lim _{k \rightarrow \infty}\left\|H\left(x^{k}\right)\right\|=\lim _{k \rightarrow \infty} \epsilon_{k}\|e\|=0 .
$$

Hence $\bar{x}$ is a solution $x^{*}$ of $H(x)=0$.

Example 2.1. Consider $n=1, M=0$, and $\phi(x)=\prod_{[0, \sqrt{\alpha}]}(\sqrt{x})+\max (\beta, x)$, where $0<\alpha<\beta$. Assumptions of Theorem 2.2 hold for this example. However, $F(x)=M x+\phi(x)$ is not a uniformly P-function, since for any $x, y \in[\alpha, \beta]$, $(x-y)(F(x)-F(y))=0$. 
From the proof of Theorem 2.2, we can immediately get the following corollary.

Corollary 2.1. Assume that $M$ is an $R_{0}$-matrix and

$$
\lim _{x_{i} \rightarrow \infty} \frac{\phi_{i}\left(x_{i}\right)-\phi_{i}(0)}{x_{i}}=0, \quad i=1,2, \ldots, n .
$$

If (2.1) with $\epsilon_{k}$ has a solution $x^{k}$, then the sequence $\left\{x^{k}\right\}$ is bounded, and any accumulation point of $\left\{x^{k}\right\}$ is a solution of $H(x)=0$.

\section{ERROR BOUNDS}

In practical applications, it is very important to know the accuracy of a computed solution. Error bounds and numerical verification methods for complementarity problems have been studied in [1, 3, 9, 10]. In this section, we give error bounds for the $\operatorname{NCP}(F)$ and its regularization problems. The error bounds are based on the observation [3] that for every $x, y, u, v \in R^{n}$,

(3.1) $\min \left(x_{i}, y_{i}\right)-\min \left(u_{i}, v_{i}\right)=\left(1-w_{i}\right)\left(x_{i}-u_{i}\right)+w_{i}\left(y_{i}-v_{i}\right), \quad i=1,2, \ldots, n$, where

$$
w_{i}= \begin{cases}0 & \text { if } y_{i} \geq x_{i}, v_{i} \geq u_{i} \\ 1 & \text { if } y_{i} \leq x_{i}, v_{i} \leq u_{i} \\ \frac{\min \left(x_{i}, y_{i}\right)-\min \left(u_{i}, v_{i}\right)+u_{i}-x_{i}}{y_{i}-v_{i}+u_{i}-x_{i}} & \text { otherwise. }\end{cases}
$$

Moreover, we have $w_{i} \in[0,1]$. Hence putting $y=M x+\phi(x)$ and $v=M u+\phi(u)$ in (3.1), we obtain the following lemma.

Lemma 3.1. For any $x, u \in R^{n}$, there is a diagonal matrix $W=\operatorname{diag}\left(w_{i}\right)$ with $w_{i} \in[0,1]$ such that

$$
H(x)-H(u)=(I-W+W M)(x-u)+W(\phi(x)-\phi(u)) .
$$

We say that the $\operatorname{NCP}(F)$ satisfies the strictly complementarity condition at a solution $x^{*}$ of (1.1) if

$$
x^{*}+F\left(x^{*}\right)>0 .
$$

Theorem 3.1. Assume that $M$ is a P-matrix and $\phi$ is a monotonically increasing and continuous diagonal function. Suppose that the strictly complementarity condition holds at the solution $x^{*}$ of (1.1), and for any $\mu>0$, there is $\gamma_{\mu}>0$ such that for $x \in \mathcal{S}\left(\epsilon_{0}\|e\|\right), x_{i} \geq \mu, x_{i} \neq x_{i}^{*}$, it holds that

$$
\frac{\phi_{i}\left(x_{i}\right)-\phi_{i}\left(x_{i}^{*}\right)}{x_{i}-x_{i}^{*}} \leq \gamma_{\mu} .
$$

Let $x^{k}$ again denote the solution of $H_{\epsilon_{k}}(x)=0$. Then there is a $c>0$ such that

$$
\left\|x^{k}-x^{*}\right\| \leq c\left\|H\left(x^{k}\right)\right\| \text {. }
$$

Proof. We observe that $\left\{x^{k}\right\} \subset \mathcal{S}\left(\epsilon_{0}\|e\|\right)$, since $H\left(x^{k}\right)=\epsilon_{k} e$ and $\epsilon_{k} \leq \epsilon_{0}$.

Let us denote

$$
\mathcal{J}=\left\{i \mid x_{i}^{*}>0\right\} \quad \text { and } \quad \mathcal{K}=\left\{i \mid x_{i}^{*}=0\right\} .
$$

By the assumption of the strictly complementarity condition, $\mathcal{J} \cap \mathcal{K}=\emptyset$. Moreover, by the continuity of $H$, there are a neighbourhood $\mathcal{B}$ of $x^{*}$ and a positive constant $\alpha$ such that for all $x \in \mathcal{B}$

$$
x_{i}<\alpha, \quad(M x+\phi(x))_{i} \geq \alpha, \quad i \in \mathcal{K},
$$


and

$$
(M x+\phi(x))_{i}<\alpha, \quad x_{i} \geq \alpha, \quad i \in \mathcal{J} .
$$

By Theorem 2.1, there is a positive integer $k_{0}>0$ such that for all $k \geq k_{0}$, we have $\epsilon_{k}<\alpha$ and $x^{k} \in \mathcal{B}$. This implies that for $k \geq k_{0}$,

$$
x_{i}^{k}>\left(M x^{k}+\phi\left(x^{k}\right)\right)_{i}, \quad i \in \mathcal{J},
$$

and

Therefore, we deduce

$$
x_{i}^{k}<\left(M x^{k}+\phi\left(x^{k}\right)\right)_{i}, \quad i \in \mathcal{K} .
$$

$$
\begin{gathered}
H_{i}\left(x^{k}\right)-H_{i}\left(x^{*}\right)=x_{i}^{k}-x_{i}^{*}, \quad i \in \mathcal{K}, \\
H_{i}\left(x^{k}\right)-H_{i}\left(x^{*}\right)=\left(M\left(x^{k}-x^{*}\right)\right)_{i}+\frac{\phi_{i}\left(x_{i}^{k}\right)-\phi_{i}\left(x_{i}^{*}\right)}{x_{i}^{k}-x_{i}^{*}}\left(x_{i}^{k}-x_{i}^{*}\right), \quad i \in \mathcal{J}, x_{i}^{k} \neq x_{i}^{*},
\end{gathered}
$$

and

$$
H_{i}\left(x^{k}\right)-H_{i}\left(x^{*}\right)=\left(M\left(x^{k}-x^{*}\right)\right)_{i}, \quad i \in \mathcal{J}, x_{i}^{k}=x_{i}^{*} .
$$

Note that assumption (3.3) implies that for $k \geq k_{0}$ and $i \in \mathcal{J}, x_{i}^{k} \neq x_{i}^{*}$, there is a $\gamma_{\alpha}$ such that

Therefore, for $k \geq k_{0}$, we have

$$
0 \leq \frac{\phi_{i}\left(x_{i}^{k}\right)-\phi_{i}\left(x_{i}^{*}\right)}{x_{i}^{k}-x_{i}^{*}} \leq \gamma_{\alpha} .
$$

$$
H\left(x^{k}\right)-H\left(x^{*}\right)=\left(I-D^{*}+D^{*}\left(M+T^{k}\right)\right)\left(x^{k}-x^{*}\right),
$$

where $D^{*}=\operatorname{diag}\left(d_{i}^{*}\right)$ and $T^{k}=\operatorname{diag}\left(t_{i}^{k}\right)$ with

$$
d_{i}^{*}= \begin{cases}1, & i \in \mathcal{J}, \\ 0, & i \in \mathcal{K}\end{cases}
$$

and

$$
t_{i}^{k}= \begin{cases}\frac{\phi_{i}\left(x_{i}^{k}\right)-\phi_{i}\left(x_{i}^{*}\right)}{x_{i}^{k}-x_{i}^{*}}, & i \in \mathcal{J}, x_{i}^{k} \neq x_{i}^{*}, \\ 0 & \text { otherwise. }\end{cases}
$$

Since $M$ is a $\mathrm{P}$-matrix and $T^{k}$ is a nonnegative diagonal matrix, the matrix $M+T^{k}$ is a $\mathrm{P}$-matrix. It is known that a matrix $A$ is a $\mathrm{P}$-matrix if and only if $I-W+W A$ is nonsingular for any diagonal matrix $W$ with $0 \leq w_{i} \leq 1$ [7]. Hence, for all $k \geq k_{0}$, the matrix $I-D^{*}+D^{*}\left(M+T^{k}\right)$ is nonsingular. Moreover by the continuity of the norm with respect to the elements of the matrix, there is a $c_{1}>0$ such that for $k \geq k_{0}$,

$$
\left\|x^{k}-x^{*}\right\| \leq \max _{t^{k} \in\left[0, \gamma_{\alpha}\right]^{n}}\left\|\left(I-D^{*}+D^{*}\left(M+T^{k}\right)\right)^{-1}\right\|\left\|H\left(x^{k}\right)\right\| \leq c_{1}\left\|H\left(x^{k}\right)\right\| .
$$

Now we consider $k \leq k_{0}$. Since $\epsilon_{k} \geq \epsilon_{k_{0}}$ for $k \leq k_{0}$, we have $x^{k} \geq \epsilon_{k_{0}} e$ for $k \leq k_{0}$. Let $S^{k}=\operatorname{diag}\left(s_{i}^{k}\right)$ with

$$
s_{i}^{k}= \begin{cases}\frac{\phi_{i}\left(x_{i}^{k}\right)-\phi_{i}\left(x_{i}^{*}\right)}{x_{i}^{k}-x_{i}^{*}}, & x_{i}^{k} \neq x_{i}^{*}, \\ 0 & \text { otherwise. }\end{cases}
$$

By the assumption of this theorem, we have $s_{i}^{k} \in\left[0, \gamma_{\epsilon_{k_{0}}}\right]$. Using (3.2), for $k \leq k_{0}$, we can write

$$
H\left(x^{k}\right)-H\left(x^{*}\right)=\left(I-W^{k}+W^{k}\left(M+S^{k}\right)\right)\left(x^{k}-x^{*}\right),
$$

where $W^{k}=\operatorname{diag}\left(w_{i}^{k}\right)$ with $w_{i}^{k} \in[0,1]$. 
Note that $M$ and $M+S^{k}$ are P-matrices. We find $\left\|x^{k}-x^{*}\right\| \leq \max _{s^{k} \in\left[0, \gamma_{\epsilon_{0}}\right]^{n}} \max _{w^{k} \in[0,1]^{n}}\left\|\left(I-W^{k}+W^{k}\left(M+S^{k}\right)\right)^{-1}\right\|\left\|H\left(x^{k}\right)\right\| \leq c_{2}\left\|H\left(x^{k}\right)\right\|$, where $s^{k}=\left(s_{i}^{k}\right)$. Let $c=\max \left(c_{1}, c_{2}\right)$. We obtain the estimate (3.4).

It is known that an H-matrix with positive diagonals is a P-matrix. In the following, we give a computable error bound of a nonnegative approximate solution of (2.1) for $M$ being an H-matrix with positive diagonals.

Lemma 3.2 (3]). Suppose that $A$ is an H-matrix with positive diagonals. Then for any diagonal matrix $W=\operatorname{diag}\left(w_{i}\right)$ with $w_{i} \in[0,1]$ the matrix $I-W+W A$ is an H-matrix with positive diagonal elements and

$$
\left|(I-W+W A)^{-1}\right| \leq \tilde{A}^{-1} \max (\Delta, I),
$$

where $\tilde{A}$ is the comparison matrix of $A$ and $\Delta$ is the diagonal part of $A$.

Theorem 3.2. Suppose that $M$ is an H-matrix with positive diagonals. Then for any $x \geq 0$, and $\epsilon_{k} \geq 0$, we have

$$
\left|x-x^{k}\right| \leq \tilde{M}^{-1} \max (D, I)\left|H_{\epsilon_{k}}(x)\right|,
$$

where $D$ is the diagonal part of $M$ and $\tilde{M}$ is the comparison matrix of $M$ and we set $H_{\epsilon_{k}}(x)=H(x)$ if $\epsilon_{k}=0$.

Proof. For a fixed $x$, let $V^{k}=\operatorname{diag}\left(v_{i}^{k}\right)$ with

$$
v_{i}^{k}:= \begin{cases}\frac{\phi_{i}\left(x_{i}\right)-\phi_{i}\left(x^{k}\right)}{x_{i}-x_{i}^{k}}, & x_{i} \neq x_{i}^{k}, \\ 0 & \text { otherwise. }\end{cases}
$$

By Lemma 3.1 and the definition of $V^{k}$, there is a diagonal matrix $W^{k}=\operatorname{diag}\left(w_{i}^{k}\right)$ with $w_{i}^{k} \in[0,1]$ such that

$H_{\epsilon_{k}}(x)=H_{\epsilon_{k}}(x)-H_{\epsilon_{k}}\left(x^{k}\right)=H(x)-H\left(x^{k}\right)=\left(I-W^{k}+W^{k}\left(M+V^{k}\right)\right)\left(x-x^{k}\right)$.

Since $\phi$ is monotonically increasing, $v_{i}^{k} \geq 0$ for $i=1,2, \ldots, n$. This implies $M+V^{k}$ is an H-matrix with positive diagonals. Hence, $I-W^{k}+W^{k}\left(M+V^{k}\right)$ is also an $\mathrm{H}$-matrix with positive diagonals, and

$$
\left|x-x^{k}\right| \leq\left|\left(I-W^{k}+W^{k}\left(M+V^{k}\right)\right)^{-1}\right|\left|H_{\epsilon_{k}}(x)\right| .
$$

By Lemma 3.2, we deduce that

$$
\left|x-x^{k}\right| \leq\left(\tilde{M}+V^{k}\right)^{-1} \max \left(D+V^{k}, I\right)\left|H_{\epsilon_{k}}(x)\right| .
$$

Let $|B|=D-\tilde{M}$. Then we have

$$
\left(\tilde{M}+V^{k}\right)^{-1}=\left(I-\left(D+V^{k}\right)^{-1}|B|\right)^{-1}\left(D+V^{k}\right)^{-1} .
$$

Since $0 \leq\left(D+V^{k}\right)^{-1}|B| \leq D^{-1}|B|$, the spectral radius $\rho\left(\left(D+V^{k}\right)^{-1}|B|\right) \leq$ $\rho\left(D^{-1}|B|\right)<1$. See [12]. Hence we can estimate the inverse of $I-\left(D+V^{k}\right)^{-1}|B|$ as follows:

$$
\begin{aligned}
\left(I-\left(D+V^{k}\right)^{-1}|B|\right)^{-1} & =I+\left(D+V^{k}\right)^{-1}|B|+\left(\left(D+V^{k}\right)^{-1}|B|\right)^{2}+\ldots \\
& \leq I+D^{-1}|B|+\left(D^{-1}|B|\right)^{2}+\ldots \\
& =\left(I-D^{-1}|B|\right)^{-1} .
\end{aligned}
$$


Therefore, we have

$$
\begin{aligned}
\left(\tilde{M}+V^{k}\right)^{-1} \max \left(D+V^{k}, I\right) & =\left(I-\left(D+V^{k}\right)^{-1}|B|^{-1}\right) \max \left(I,\left(D+V^{k}\right)^{-1}\right) \\
& \leq\left(I-D^{-1}|B|\right)^{-1} \max \left(I, D^{-1}\right) \\
& =\tilde{M}^{-1} \max (D, I) .
\end{aligned}
$$

We complete the proof.

Remark 3.1. Let $x^{*}$ be the solution of the non-Lipschitzian $\operatorname{NCP}(F)$. Since $H_{0}(x)=$ $H(x)$, Theorem 3.2 yields a componentwise error bound for any $x \geq 0$,

$$
\left|x-x^{*}\right| \leq \tilde{M}^{-1} \max (D, I)|H(x)| .
$$

Moreover, for any monotone vector norm and the corresponding operator norm, we have

$$
\left\|x-x^{*}\right\| \leq\left\|\tilde{M}^{-1} \max (D, I)\right\|\|H(x)\| .
$$

(We say $\|\cdot\|$ is a monotone vector norm if for any $x, y \in R^{n},|x| \leq|y|$ implies that $\|x\| \leq\|y\|$, which is equivalent to $\|x\|=\||x|\|$. Any $p$-norm $(p \geq 1)$ is a monotone vector norm. See [8].)

\section{Projection methods}

In this section we consider a projection method for the solution of (2.1). We use Theorem 3.2 to construct an interval vector, which contains the solution of (2.1). Starting from a point in the interval, we generate a sequence by the projection method. We prove that the sequence converges to the solution of (2.1). Using (3.5) or (3.6), we can provide error bounds of an approximate solution to the exact solution of (1.1).

By rewriting (2.1) as in the proof of Lemma 2.1, we obtain the following NCP:

$$
\min (y, M y+\phi(y+\epsilon e)+\epsilon(M-I) e)=0,
$$

where $y=x-\epsilon e$. By Lemma 2.1, if $M$ is a P-matrix, then (4.1) has a unique solution $y^{*} \geq 0$, and thus (2.1) has a unique solution $x_{\epsilon}^{*}=y^{*}+\epsilon e>0$. Defining a diagonal function

$$
\psi(y):=\phi(y+\epsilon e)+\epsilon(M-I) e,
$$

(4.1) can be written as

$$
\min (y, M y+\psi(y))=0 .
$$

Here $\psi$ is a monotonically increasing and continuous diagonal function. Furthermore (4.2) can be rewritten as a fixed point problem

$$
y=\max (0, y-\Lambda(M y+\psi(y))),
$$

where $\Lambda$ is a diagonal matrix with positive diagonal elements.

For all $u, v$ contained in some interval vector $[y]$ we have

$$
\psi(u)-\psi(v)=\delta \psi(u, v)(u-v),
$$

where the diagonal matrix $\delta \psi(u, v)$ is the slope of $\psi$ for $u$ and $v$. Assume that for all $u, v, \in[y]$, the slope $\delta \psi(u, v)$ can be bounded by some diagonal interval matrix,

$$
\delta \psi(u, v) \in[\underline{\delta}, \bar{\delta}], u, v \in[y] .
$$


Since $\psi$ is a monotonically increasing diagonal function, we can assume that $\underline{\delta} \geq 0$. We assume that the bounds for the slope behave inclusion monotone:

$$
[y] \subseteq[z] \quad \Rightarrow \quad[\underline{\delta}, \bar{\delta}]_{[y]} \subseteq[\underline{\delta}, \bar{\delta}]_{[z]},
$$

where the indices $[y]$ and $[z]$ indicate that the bounds for the slope belong to the corresponding intervals $[y]$ and $[z]$. This inclusion monotonicity is a natural assumption.

Let $D$ be the diagonal part of $M$ and $B=D-M$. Let $\delta$ be a diagonal matrix with positive diagonals. Set

$$
\Lambda=\omega(D+\delta)^{-1}
$$

with $\omega>0$ in (4.3). We find that $y^{*}$ solves (4.2) if and only if $y^{*}$ is a fixed point of the following equations:

$$
y=\max \left\{0, \omega(D+\delta)^{-1}\left(R y+S y+\delta y-\psi(y)+\frac{1-\omega}{\omega}(D+\delta) y\right)\right\},
$$

where matrices $R$ and $S$ satisfy $B=R+S$ and $|B|=|R|+|S|$.

Now we propose a projection method for solving (4.2).

\section{Projection Method.}

Initial Step. Choose $y^{0} \geq 0$ and compute the nonnegative matrix $C:=$ $\tilde{M}^{-1} \max (D, I)$.

Set a vector

$$
r^{0}:=C\left|\min \left(y^{0}, M y^{0}+\psi\left(y^{0}\right)\right)\right|
$$

and an interval vector

$$
\left[y^{0}\right]=\left[y^{0}-r^{0}, y^{0}+r^{0}\right] \cap R_{+}^{n} . \quad(\text { See Remark 4.1.) }
$$

Iteration. For $l=0,1,2, \ldots$

Step 1. Compute $\left[\underline{\delta}^{l}, \bar{\delta}^{l}\right]$, such that for all $u, v \in\left[y^{l}\right]$

$$
\psi(u)-\psi(v) \in\left[\underline{\delta}^{l}, \bar{\delta}^{l}\right](u-v) .
$$

Step 2. Compute $\hat{y}^{l+1}$. (See Remark 4.2.)

Step 3. Compute $\left[y^{l+1}\right]$ (with the property $\left[y^{l+1}\right] \subseteq\left[y^{l}\right]$ and $y^{*} \in\left[y^{l+1}\right]$ ). (See Remark 4.3.)

Step 4. Compute the projection

$$
y^{l+1}=\Pi_{\left[y^{l+1}\right]}\left(\hat{y}^{l+1}\right) .
$$

Remark 4.1. By Remark 3.1, the initial interval vector $\left[y^{0}\right]$ contains a solution $y^{*}$ of (4.2), if $M$ is an H-matrix with positive diagonal elements.

Remark 4.2. $\hat{y}^{l+1}$ in Step 2 is computed by

$$
\hat{y}^{l+1}=\max \left\{0, \omega\left(D+\bar{\delta}^{l}\right)^{-1}\left(R \hat{y}^{l+1}+S y^{l}+\bar{\delta}^{l} y^{l}-\psi\left(y^{l}\right)+\frac{1-\omega}{\omega}\left(D+\bar{\delta}^{l}\right) y^{l}\right)\right\}
$$

which includes the following two cases:

(i) Relaxed Jacobi-like method: $R=0, S=D-M$. 
(ii) Successive overrelaxation-like method: $R=L, S=U$, where $-L$ and $-U$ denote the strictly lower and strictly upper triangular part of $M$, respectively.

Case (i) contains a Jacobi-like method as a special case $(\omega=1)$. Moreover, case (ii) contains a Gauss-Seidel-like method as a special case $(\omega=1)$. In general, any splitting method for which the spectral radius $\rho\left(\mathcal{L}_{\omega}^{l}\right)<\sigma_{1}<1$ can be used for the computation of $\hat{y}^{l+1}$ where

$$
\mathcal{L}_{\omega}^{l}=\left(D+\bar{\delta}^{l}-\omega|R|\right)^{-1}\left(\omega|S|+\omega\left(\bar{\delta}^{l}-\underline{\delta}^{l}\right)+(1-\omega)\left(D+\bar{\delta}^{l}\right)\right) .
$$

Remark 4.3. The simplest choice in Step 3 is $\left[y^{l+1}\right]=\left[y^{l}\right]$, which means $\left[y^{l}\right]=\left[y^{0}\right]$, for all $l=1,2, \ldots$. However, one can also spend some work for the calculation of $\left[y^{l+1}\right]$ by two additional steps:

$$
\begin{array}{ll}
\text { Step 3a. } & r^{l+1}=C\left|\min \left(\hat{y}^{l+1}, M \hat{y}^{l+1}+\psi\left(\hat{y}^{l+1}\right)\right)\right| . \\
\text { Step 3b. } & {\left[y^{l+1}\right]=\left[\hat{y}^{l+1}-r^{l+1}, \hat{y}^{l+1}+r^{l+1}\right] \cap\left[y^{l}\right] .}
\end{array}
$$

By Remark 3.1, and $y^{*} \in\left[y^{0}\right]$, we have $y^{*} \in\left[y^{l}\right]$ for all $l \geq 0$. Moreover, one can also use (3.6) with the infinity norm for the calculation of $\left[y^{l+1}\right]$. In particular, we can replace Step 3a by

$$
r^{l+1}=\|C\|_{\infty}\left\|\min \left(\hat{y}^{l+1}, M \hat{y}^{l+1}+\psi\left(\hat{y}^{l+1}\right)\right)\right\|_{\infty} e .
$$

If $\tilde{M}^{-1} \geq 0$, then $C=\tilde{M}^{-1} \max (D, I) \geq 0$. Since $\|C\|_{\infty}=\|C e\|_{\infty}=:\|v\|_{\infty}$, we can compute $\|C\|_{\infty}$ by solving a system of linear equations, $\tilde{M} v=\max (D, I) e$.

Now we study the convergence of the projection method for $M$ being an $H$ matrix with positive diagonals.

The projection method generates a sequence $\left\{y^{l}\right\}$. We will show that the sequence $y^{l}$ will converge to $y^{*}$.

Note that $\bar{\delta}^{l} \geq 0$ since $\psi$ is monotonically increasing. Moreover, we have $y^{*} \in\left[y^{l}\right]$ for all $l \geq 0$.

By the definition of $\left[y^{0}\right]$ combined with Step $3 \mathrm{~b}$, we have $y^{l} \geq 0$ and thus $\left[y^{l}\right]=$ $\left[\underline{y}^{l}, \bar{y}^{l}\right] \subseteq R_{+}^{n}$ for all $l \geq 0$. By the substitution $y=x-\epsilon e$, the value $x_{i}=0$ corresponds to $y_{i}=-\epsilon<0$. Hence the slope of $\psi_{i}$ is bounded for each interval $\left[y_{i}\right]=\left[\underline{y}_{i}, \bar{y}_{i}\right]$ with lower bound $\underline{y}_{i} \geq 0$. This means

$$
\left[\underline{\delta}^{l}, \bar{\delta}^{l}\right] \subseteq\left[\delta_{1}, \delta_{2}\right]
$$

where $\left[\delta_{1}, \delta_{2}\right]$ is a fixed interval diagonal matrix. We can assume $\delta_{1} \geq 0$ since all $\psi_{i}$ are monotonically increasing.

It is easy to see that for any $x, y \in R^{n},|\max (0, x)-\max (0, y)| \leq|x-y|$. Hence, from (4.4) with $\delta=\bar{\delta}$ and (4.5), we have

$$
\begin{aligned}
\left|\hat{y}^{l+1}-y^{*}\right| \leq \omega\left(D+\bar{\delta}^{l}\right)^{-1} & \left(|R|\left|\hat{y}^{l+1}-y^{*}\right|+|S|\left|y^{l}-y^{*}\right|\right. \\
& \left.+\left|\bar{\delta}^{l}+\frac{1-\omega}{\omega}\left(D+\bar{\delta}^{l}\right)-\delta \psi\left(y^{l}, y^{*}\right)\right|\left|y^{l}-y^{*}\right|\right) .
\end{aligned}
$$

Since $\delta \psi\left(y^{l}, y^{*}\right) \in\left[\underline{\delta}^{l}, \bar{\delta}^{l}\right]$, we have for $0<\omega \leq 1$,

$$
\left|\bar{\delta}^{l}+\frac{1-\omega}{\omega}\left(D+\bar{\delta}^{l}\right)-\delta \psi\left(y^{l}, y^{*}\right)\right| \leq \bar{\delta}^{l}-\underline{\delta}^{l}+\frac{1-\omega}{\omega}(D+\bar{\delta}) .
$$


Therefore, we obtain

$$
\left(\left(D+\bar{\delta}^{l}\right)-\omega|R|\right)\left|\hat{y}^{l+1}-y^{*}\right| \leq\left(\omega|S|+\omega\left(\bar{\delta}^{l}-\underline{\delta}^{l}\right)+(1-\omega)\left(D+\bar{\delta}^{l}\right)\right)\left|y^{l}-y^{*}\right| .
$$

By Step 4 and the property of the projection of $\hat{y}^{l+1}$ onto the interval $\left[y^{l+1}\right]$ which contains the solution $y^{*}$, we have

$$
\left|y^{l+1}-y^{*}\right| \leq\left|\hat{y}^{l+1}-y^{*}\right| .
$$

Therefore, we obtain

$$
\left|y^{l+1}-y^{*}\right| \leq\left(D+\bar{\delta}^{l}-\omega|R|\right)^{-1}\left(\omega|S|+\omega\left(\bar{\delta}^{l}-\underline{\delta}^{l}\right)+(1-\omega)(D+\bar{\delta})\right)\left|y^{l}-y^{*}\right|,
$$

provided $\left(D+\bar{\delta}^{l}-\omega|R|\right)^{-1}$ exists and is nonnegative, which is obviously true for the two cases in Remark 4.2.

Since the sequence $\left\{\left[\underline{\delta}^{l}, \bar{\delta}^{l}\right]\right\}$ converges to $\left[\underline{\delta}^{*}, \bar{\delta}^{*}\right]$, it follows that for

$$
\mathcal{L}_{\omega}^{l}:=\left(D+\bar{\delta}^{l}-\omega|R|\right)^{-1}\left(\omega|S|+\omega\left(\bar{\delta}^{l}-\underline{\delta}^{l}\right)+(1-\omega)\left(D+\bar{\delta}^{l}\right)\right)
$$

we have

$$
\lim _{l \rightarrow \infty} \mathcal{L}_{\omega}^{l}=\mathcal{L}_{\omega}^{*}
$$

where

$$
\mathcal{L}_{\omega}^{*}=\left(D+\bar{\delta}^{*}-\omega|R|\right)^{-1}\left(\omega|S|+\omega\left(\bar{\delta}^{*}-\underline{\delta}^{*}\right)+(1-\omega)\left(D+\bar{\delta}^{*}\right)\right) .
$$

Now we show that under certain conditions, which hold especially for the two cases in Remark 4.2, $\rho\left(\mathcal{L}_{\omega}^{*}\right)<1$. Consider the matrix $A=P-Q$ with

$$
P=\frac{1}{\omega}\left(D+\bar{\delta}^{*}-\omega|R|\right)
$$

and

$$
Q=\frac{1}{\omega}\left(\omega|S|+\omega\left(\bar{\delta}^{*}-\underline{\delta}^{*}\right)+(1-\omega)\left(D+\bar{\delta}^{*}\right)\right) .
$$

It is easy to verify

$$
\begin{aligned}
A=P-Q & =\frac{1}{\omega}\left(\omega\left(D+\bar{\delta}^{*}\right)-\omega|R|-\omega|S|-\omega \bar{\delta}^{*}+\omega \underline{\delta}^{*}\right) \\
& =D+\underline{\delta}^{*}-|R|-|S|=D+\underline{\delta}^{*}-|B| .
\end{aligned}
$$

Since $\underline{\delta}^{*} \geq 0$ and $D-|B|$ is an M-matrix, $A$ is also an M-matrix, and therefore $A^{-1}=\left(D+\underline{\delta}^{*}-|R|-|S|\right)^{-1} \geq 0$. Furthermore, $P^{-1} \geq 0$ and $Q \geq 0$. Therefore, $A=P-Q$ is a regular splitting of $A$, which implies

$$
\rho\left(\mathcal{L}_{\omega}^{*}\right)=\rho\left(P^{-1} Q\right)<1
$$

See 12$]$.

Using (4.6), we have

$$
\left|y^{l+1}-y^{*}\right| \leq\left(\prod_{i=1}^{l} \mathcal{L}_{\omega}^{i}\right)\left|y^{0}-y^{*}\right| .
$$

Since the spectral radius $\rho\left(\mathcal{L}_{\omega}^{*}\right)<1$, for any given $\sigma \in\left(0,1-\rho\left(\mathcal{L}_{\omega}^{*}\right)\right)$, there exists a matrix norm such that $\left\|\mathcal{L}_{\omega}^{*}\right\| \leq \rho\left(\mathcal{L}_{\omega}^{*}\right)+\sigma<1$. By the continuity of the norm, there exist an $L>0$ and $\sigma_{1} \in\left(\rho\left(\mathcal{L}_{\omega}^{*}\right)+\sigma, 1\right)$, such that for all $l \geq L$,

$$
\left\|\mathcal{L}_{\omega}^{l}\right\| \leq \sigma_{1}<1 .
$$


Therefore for $l>L$, we have

$$
\left\|\prod_{i=1}^{l} \mathcal{L}_{\omega}^{i}\right\| \leq\left\|\prod_{i=1}^{L-1} \mathcal{L}_{\omega}^{i}\right\|\left\|\prod_{i=L}^{l} \mathcal{L}_{\omega}^{i}\right\| \leq c \sigma_{1}^{l-L+1},
$$

where

$$
c=\left\|\prod_{i=1}^{L-1} \mathcal{L}_{\omega}^{i}\right\| .
$$

Therefore, we deduce that

$$
\lim _{l \rightarrow \infty}\left\|\prod_{i=1}^{l} \mathcal{L}_{\omega}^{i}\right\|=0
$$

which is equivalent to

$$
\lim _{l \rightarrow \infty} \prod_{i=1}^{l} \mathcal{L}_{\omega}^{i}=0 .
$$

From (4.8), we find that $\lim _{l \rightarrow \infty} y^{l}=y^{*}$. Moreover, we have for $l \geq L$,

$$
\left\|y^{l+1}-y^{*}\right\| \leq \sigma_{1}\left\|y^{l}-y^{*}\right\| .
$$

This means that the sequence $\left\{y^{l}\right\}$ is convergent to $y^{*}$ linearly. We summarize our analysis as follows.

Theorem 4.1. If $M$ is an H-matrix with positive diagonals, then the projection method with $M=D-B=D-R-S$ is convergent to the solution $y^{*}$ of (4.2) for any initial point $y^{0} \geq 0$, provided that $0<\omega \leq 1$ and $R$ is chosen such that $(D-\omega|R|)^{-1}$ exists and is nonnegative. Furthermore, the convergence is at least linear.

Remark 4.4. The spectral radius is continuously dependent on the matrix elements. It is clear that there is an $\omega_{1}>1$ such that for all $\omega \in\left(1, \omega_{1}\right)$ Theorem 4.1 also holds.

Remark 4.5. The projection method also generates a sequence of intervals which satisfies

$$
y^{*} \in\left[y^{l+1}\right] \subseteq\left[y^{l}\right]
$$

The sequence is therefore convergent to some interval vector $\left[y^{*}\right]$. From the inequalities

$$
\left|y^{l+1}-y^{*}\right| \leq\left|\hat{y}^{l+1}-y^{*}\right| \leq\left|y^{l}-y^{*}\right|
$$

the convergence of $\left\{y^{l}\right\}$ to $y^{*}$ implies that the radius $r^{*}$ of the interval $\left[y^{*}\right]$ is zero if we use Steps $3 \mathrm{a}$ and $3 \mathrm{~b}$ in Remark 4.3, which means that $\left[y^{*}\right]=y^{*}$.

Remark 4.6. The projection method provides intervals containing the solution $x^{*}$ of the non-Lipschitzian $\mathrm{NCP}(F)$. In particular, if $M$ is an H-matrix with positive diagonals, from

$$
\begin{gathered}
x_{\epsilon}^{*}-\epsilon e=y^{*} \in\left[y^{l}\right], \\
H\left(x_{\epsilon}^{*}\right)=H\left(x_{\epsilon}^{*}\right)-H_{\epsilon}\left(x_{\epsilon}^{*}\right)=\epsilon e
\end{gathered}
$$

and

$$
\left|x_{\epsilon}^{*}-x^{*}\right| \leq \tilde{M}^{-1} \max (D, I)\left|H\left(x_{\epsilon}^{*}\right)\right|=C \epsilon e
$$

we can easily find that for all $l \geq 0$,

$$
x^{*} \in\left[y^{l}\right]+\epsilon[(I-C) e,(I+C) e] .
$$


We can also use Remark 3.1 to get

$$
x^{*} \in\left[\hat{y}^{l}-C\left|H\left(y^{l}\right)\right|, \hat{y}^{l}+C\left|H\left(y^{l}\right)\right|\right] .
$$

\section{Numerical EXPERIMENTS}

We have done very extensive numerical experiments. Numerical results show that the regularized projection method is efficient to solve the non-Lipschitzian complementarity problems. Moreover, the error bounds (4.9) and (4.10) provide computable error estimation for verifying the accuracy of a computed solution.

We report numerical results for a free boundary problem in [2.

Example 5.1. Let $\Omega=(0,1) \times(0,1)$, and let $p \in(0,1)$. We consider

$$
\begin{array}{cl}
-\triangle u+\frac{9}{(1-p)^{2}} u^{p}=f(z) & \text { in } \Omega_{+}, \\
u=0 & \text { in } \Omega_{0}, \\
u=|\nabla u|=0 & \text { on } \Gamma, \\
u=g(z) & \text { on } \partial \Omega,
\end{array}
$$

where $\Omega_{+}=\{z \in \Omega \mid u(z)>0\}, \Omega_{0}=\{z \in \Omega \mid u(z)=0\}$, and $\Gamma=\partial \Omega_{0}=\partial \Omega_{+} \cap \Omega$ are unknown. Let $r^{2}=z_{1}^{2}+z_{2}^{2}$. We choose

$$
f(z)=\frac{9}{r(1-p)^{2}}\left(\frac{3 r-1}{2}\right)^{\frac{2 p}{1-p}} \max \left(0, r-\frac{1}{3}\right)
$$

and

$$
g(z)=\left(\frac{3 r-1}{2}\right)^{\frac{2}{1-p}} \max \left(0, r-\frac{1}{3}\right), \quad z \in \partial \Omega .
$$

This problem has a unique solution

$$
u(z)=\left(\frac{3 r-1}{2}\right)^{\frac{2}{1-p}} \max \left(0, r-\frac{1}{3}\right) .
$$

Using the five-point finite difference approximation, we obtain a nonlinear complementarity problem with $F(x)=M x+\phi(x)$, where $M$ is an M-matrix,

$$
\phi(x)=E \max \left(0, x^{p}\right)+q,
$$

$E$ is an $n \times n$ diagonal matrix with positive diagonals and $q$ is a vector in $R^{n}$. Here the components of $x$ are the approximations to the exact solution $u(z)$ at the grid points of $\Omega$. Our experiments were performed with 900 interior points of $\Omega$.

In Table 1 and Table 2, we report some numerical results of the projection method for solving Example 5.1. We used the successive overrelaxation-like method described in (ii) of Remark 4.2. In all experiments we used the vector $y^{0}=$ $(1, \ldots, 1)^{T}$ for computing the interval vector $\left[y^{0}\right]$ in the initial step of the projection method.

In Table 1, we list iteration numbers with different relaxation parameter $\omega$ for solving

$$
H_{\epsilon_{k}}(y)=\min \left(y, M y+\epsilon_{k}(M-I) e+\phi\left(y+\epsilon_{k} e\right)\right)=0
$$

with different values of $\epsilon_{k}=2^{-k}$ and $p$. For a given value of $\epsilon_{k}$, we stopped the projection method when the following inequality holds:

$$
\left\|\hat{y}^{l+1}-\hat{y}^{l}\right\|_{\infty} \leq 10^{-13}\left\|\hat{y}^{l+1}\right\|_{\infty} .
$$


To save computation time, we set

$$
\left[y^{l+1}\right]=\left[y^{l}\right]
$$

in Step 3 of the projection method. The value $\omega_{\text {opt }}$, for which the smallest number of iterations was taken to get (5.2), was determined experimentally.

TABle 1. Number of iterations: $K_{1}$ for $\omega=1, K_{\text {opt }}$ for $\omega=\omega_{\text {opt }}$

\begin{tabular}{|c|c|c|c|c|}
\hline$p$ & $\epsilon_{k}$ & $\omega_{\text {opt }}$ & $K_{1}$ & $K_{\text {opt }}$ \\
\hline \multirow{5}{*}{0.3} & 1 & 1.8 & 1570 & 142 \\
& $2^{-5}$ & 1.8 & 1578 & 175 \\
& $2^{-10}$ & 2.1 & 2060 & 169 \\
& $2^{-15}$ & 4.6 & 7573 & 558 \\
\hline \multirow{5}{*}{0.5} & 1 & 1.7 & 822 & 111 \\
& $2^{-5}$ & 1.8 & 1022 & 126 \\
& $2^{-10}$ & 2.0 & 1251 & 128 \\
& $2^{-15}$ & 2.0 & 2694 & 847 \\
\hline \multirow{5}{*}{0.7} & 1 & 1.6 & 431 & 88 \\
& $2^{-5}$ & 1.7 & 517 & 86 \\
& $2^{-10}$ & 1.8 & 603 & 106 \\
& $2^{-15}$ & 2.0 & 842 & 187 \\
\hline \multirow{3}{*}{0.9} & 1 & 1.4 & 108 & 42 \\
& $2^{-5}$ & 1.5 & 123 & 44 \\
& $2^{-10}$ & 1.6 & 143 & 48 \\
& $2^{-15}$ & 1.8 & 171 & 45 \\
\hline
\end{tabular}

In Table 2, we show the total iteration number $N$ with a fixed relaxation parameter $\omega$ for solving (5.1) for $\epsilon_{k}=2^{-k}, k=0,2, \ldots, 30$, and error bounds of an approximation solution of (5.1) with $\epsilon_{k}=2^{-30}$ to the exact solution of the $\operatorname{NCP}(F)$. In our numerical test, if $\hat{y}^{l+1}$ satisfies (5.2), we change $\epsilon_{k}=2^{-k}$ to $\epsilon_{k}=2^{-k-2}$ and set $y^{0}=\hat{y}^{l+1}$ as the initial vector for solving (5.1) with the new $\epsilon_{k}$. The error bounds were obtained by (4.10).

TABLE 2. Error bounds $\left\|x^{*}-y^{l}\right\|_{\infty} \leq e r r b$

\begin{tabular}{|c|c|c|c|c|c|c|}
\hline$p$ & $\omega$ & $N$ & $e r r b$ & $\omega$ & $N$ & $e r r b$ \\
\hline 0.3 & 1.0 & 40030 & $3.5 e-5$ & 1.8 & 18764 & $1.9 e-5$ \\
\hline 0.5 & 1.0 & 16647 & $7.4 e-6$ & 1.8 & 6169 & $4.0 e-6$ \\
\hline 0.7 & 1.6 & 2025 & $2.1 e-6$ & 1.8 & 1643 & $1.9 e-6$ \\
\hline 0.9 & 1.6 & 442 & $5.4 e-5$ & 1.8 & 774 & $9.1 e-5$ \\
\hline
\end{tabular}

The numerical results were obtained by using the programming language PASCAL-XSC on an HP-9000 workstation in the University of Karlsruhe. 


\section{ACKNOWLEDGEMENT}

We are grateful to Dr. U. Schäfer for carefully reading this paper, and for checking the numerical results.

\section{REFERENCES}

[1] G.E. Alefeld, X. Chen and F.A. Potra, Numerical validation of solutions of complementarity problems: The nonlinear case, Numer. Math., 92 (2002) 1-16. MR1917363 (2003f:65109)

[2] J.W. Barrett and R.M. Shanahan, Finite element approximation of a model reaction-diffusion problem with a non-Lipschitz nonlinearity, Numer. Math., 59(1991) 217-242. MR.1106381 (92c:65122)

[3] X. Chen and S. Xiang, Computation of error bounds for P-matrix linear complementarity problems, Math. Programming, 106(2006) 513-525. MR2216793 (2006k:90131)

[4] R.W. Cottle, J.-S. Pang and R.E. Stone, The Linear Complementarity Problem, Academic Press, Boston, MA, 1992. MR 1150683 (93f:90001)

[5] J.E. Dennis and R.E. Schnabel, Numerical Methods for Unconstrained Optimization and Nonlinear Equations, Prentice-Hall, Englewood Cliffs, 1983. MR702023 (85j:65001)

[6] F. Facchinei and J.-S. Pang, Finite-Dimensional Variational Inequalities and Complementarity Problems I, II, Springer-Verlag, New York, 2003.

[7] S.A. Gabriel and J.J. Moré, Smoothing of mixed complementarity problems, Complementarity and Variational Problems: State of the Art, eds by M.C. Ferris and J.-S. Pang, (SIAM Publications, Philadelphia, PA, 1997), 105-116. MR1445075 (98c:90161)

[8] J.M. Ortega and W.C. Rheinboldt, Iterative Solution of Nonlinear Equations in Several Variables, Academic Press, New York, 1970. MR0273810 (42:8686)

[9] J.-S. Pang, Error bounds in mathematical programming, Math. Programming, 79(1997) 299332. MR.1464772 (98j:90075)

[10] U. Schäfer, An enclosure method for free boundary problems based on a linear complementarity problem with interval data, Numer. Func. Anal. Optim., 22(2001) 991-1011. MR1871871 (2003d:90110)

[11] P. Tseng, An infeasible path-following method for monotone complementarity problems, SIAM J. Optim., 7(1997) 386-402. MR.1443625 (98c:90169)

[12] R. Varga, Matrix Iterative Analysis, Second Revised and Expanded Edition, Springer-Verlag, New York 2000. MR:1753713 (2001g:65002)

Institute of Applied and Numerical Mathematics, University of Karlsruhe (Karlsruhe Institute of Technology KIT), D-76128 Karlsruhe, Germany

E-mail address: goetz.alefeld@math.uni-karlsruhe.de

Department of Mathematical Sciences, Hirosaki University, 036-8561 Hirosaki, Japan

E-mail address: chen@cc.hirosaki-u.ac.jp 